\title{
Dynamical properties of elemental metabolism distinguish attention deficit hyperactivity disorder from autism spectrum disorder
}

\author{
Christine Austin ${ }^{1}$, Paul Curtin ${ }^{1}$, Austen Curtin ${ }^{1}$, Chris Gennings ${ }^{1}$, Manish Arora ${ }^{1}$, Kristiina Tammimies $\mathbb{B}^{2}$, \\ Johan Isaksson ${ }^{2,3}$, Charlotte Willfors ${ }^{2,4}$ and Sven Bölte (i) 2,5
}

\begin{abstract}
Attention deficit hyperactivity disorder (ADHD) and autism spectrum disorder (ASD) are neurodevelopmental conditions of overlapping etiologies and phenotypes. For ASD, we recently reported altered elemental metabolic patterns in the form of short and irregular zinc and copper cycles. Here, we extend the application of these biomarkers of prenatal and early postnatal elemental metabolism to distinguish between individuals diagnosed with ADHD and/ or ASD and neurotypical controls. We recruited twins discordant for ADHD, ASD and other neurodevelopmental diagnoses from national twin studies in Sweden $(N=74)$ diagnosed according to DSM-5 clinical consensus and standardized psychiatric instruments. Detailed temporal profiles of exposure to 10 metals over the prenatal and early childhood periods were measured using tooth biomarkers. We used recurrence quantification analysis (RQA) to characterize properties of cyclical metabolic patterns of these metals. Regularity (determinism) and complexity (entropy) of elemental cycles was consistently reduced in ADHD for cobalt, lead, and vanadium (determinism: cobalt, $\beta=-0.03, P=0.017$; lead, $\beta=-0.03, P=0.016$; and vanadium, $\beta=-0.03, P=0.01$. Entropy: cobalt, $\beta=-0.13, P=$ 0.017 ; lead, $\beta=-0.18, P=0.016$; and vanadium, $\beta=-0.15, P=0.008$ ). Further, we found elemental pathways and dynamical features specific to ADHD vs ASD, and unique characteristics associated with ADHD/ASD combined presentation. Dysregulation of cyclical processes in elemental metabolism during prenatal and early postnatal development not only encompasses pathways shared by ADHD and ASD, but also comprise features specific to either condition.
\end{abstract}

\section{Introduction}

Attention deficit hyperactivity disorder (ADHD) is a common neurodevelopmental condition affecting an estimated $5.3 \%$ of children and $2.5 \%$ of adults

\footnotetext{
Correspondence: Christine Austin (christine.austin@mssm.edu) or Paul Curtin (paul.curtin@mssm.edu) or Sven Bölte (sven.bolte@ki.se) ${ }^{1}$ Department of Environmental Medicine and Public Health, Icahn School of Medicine at Mount Sinai, One Gustave L Levy Place, Box 1057, New York, NY 10029, USA

${ }^{2}$ Karolinska Institutet Center of Neurodevelopmental Disorders (KIND), Centre for Psychiatry Research, Department of Women's and Children's Health, Karolinska Institutet, \& Stockholm Health Care Services, Stockholm County Council, Stockholm, Sweden

Full list of author information is available at the end of the article. The members of the Emergent Dynamical Systems group (CA, PC, AC, CG, and MA) are co-first authors
}

worldwide $^{1,2}$. Heritability estimates average $75 \%$ with a polygenic liability comprising both common and rare variants, suggesting that environmental exposures are also at play $^{3-5}$. However, the role of specific environmental exposures and their metabolism in the etiology of ADHD and co-occurring neurodevelopmental conditions, such as autism spectrum disorder $(\mathrm{ASD})^{6}$, remains unclear ${ }^{7}$. Few studies have investigated the association between elemental exposures and ADHD (for reviews, $\operatorname{see}^{8}$ and $^{3}$ ). In addition, those studies have exclusively relied on crosssectional measures of exposure, primarily blood metal concentrations. They found differences between ADHD and non-ADHD controls for lead, mercury, manganese, iron, zinc, and copper ${ }^{9-18}$. Unfortunately, this research

\section{(c) The Author(s) 2019}

(c) (i) Open Access This article is licensed under a Creative Commons Attribution 4.0 International License, which permits use, sharing, adaptation, distribution and reproduction cc) in any medium or format, as long as you give appropriate credit to the original author(s) and the source, provide a link to the Creative Commons license, and indicate if changes were made. The images or other third party material in this article are included in the article's Creative Commons license, unless indicated otherwise in a credit line to the material. If material is not included in the article's Creative Commons license and your intended use is not permitted by statutory regulation or exceeds the permitted use, you will need to obtain permission directly from the copyright holder. To view a copy of this license, visit http://creativecommons.org/licenses/by/4.0/. 
does not include information on the timing of exposures relative to their effects on neurodevelopment, or permit contrasts between different neurodevelopmental disorders. For example, lead is the most widely investigated toxic exposure linked to ADHD, but study findings derived from a single time-point measurement do not resolve temporal variations in exposure during critical developmental windows ${ }^{3}$. Using novel tooth matrix biomarkers, we recently demonstrated that essential and toxic elements are differentially regulated in $\mathrm{ASD}^{19}$. This was achieved by focusing analyses on time variant cycles in elemental metabolism, which would be missed in analyses relying solely on single time-point concentrations. The combination of multiple cohorts in that study, each drawn from different sites with varying enrollment protocols, nonetheless did not allow a fine scale differentiation of ASD and ADHD phenotypes, as were characterized in the RATSS cohort.

Here, we test the hypothesis that cycles of essential and toxic elements are dysregulated during prenatal and early postnatal development in ADHD and that these profiles distinguish individuals with ADHD from individuals with ASD as well as ADHD/ASD combined presentation ${ }^{6}$. We utilize recurrence quantification analysis (RQA $)^{20-22}$, a non-linear method of characterizing signal dynamics, particularly the frequency, duration and complexity of cyclical processes in longitudinal time-series measures of elemental metabolism. In a well-characterized population-derived sample of twins in Sweden ${ }^{19,23,24}$, which includes monozygotic twins discordant for ADHD, we apply RQA to fine scale temporal profiles of metal uptake during the prenatal and early postnatal period made possible by recently developed tooth matrix biomarkers. The twin design allows us to account for underlying genetic factors, especially in monozygotic twins.

\section{Materials and methods \\ Participant characteristics}

Study samples were pulled from the Roots of Autism and ADHD Twin Study in Sweden (RATSS) ${ }^{23}$. Participant recruitment and tooth collection within RATSS have been previously described ${ }^{24}$. Briefly, we collected and analyzed teeth from 74 participants: 30 complete twin pairs, 1 triplet group, and 11 individuals from twin pairs whose sibling did not donate a tooth. For our primary analysis, we measured metals in 13 cases of ADHD, 8 ASD, 12 ADHD/ASD combined, and 41 neurotypical controls (see Table 1 for participant characteristics). This sample size

Table 1 RATSS Participant Characteristics. (A) Participant numbers, gestational age and birth weight. (B) Mean IQ (standrd deviation) by diagnostic category

A

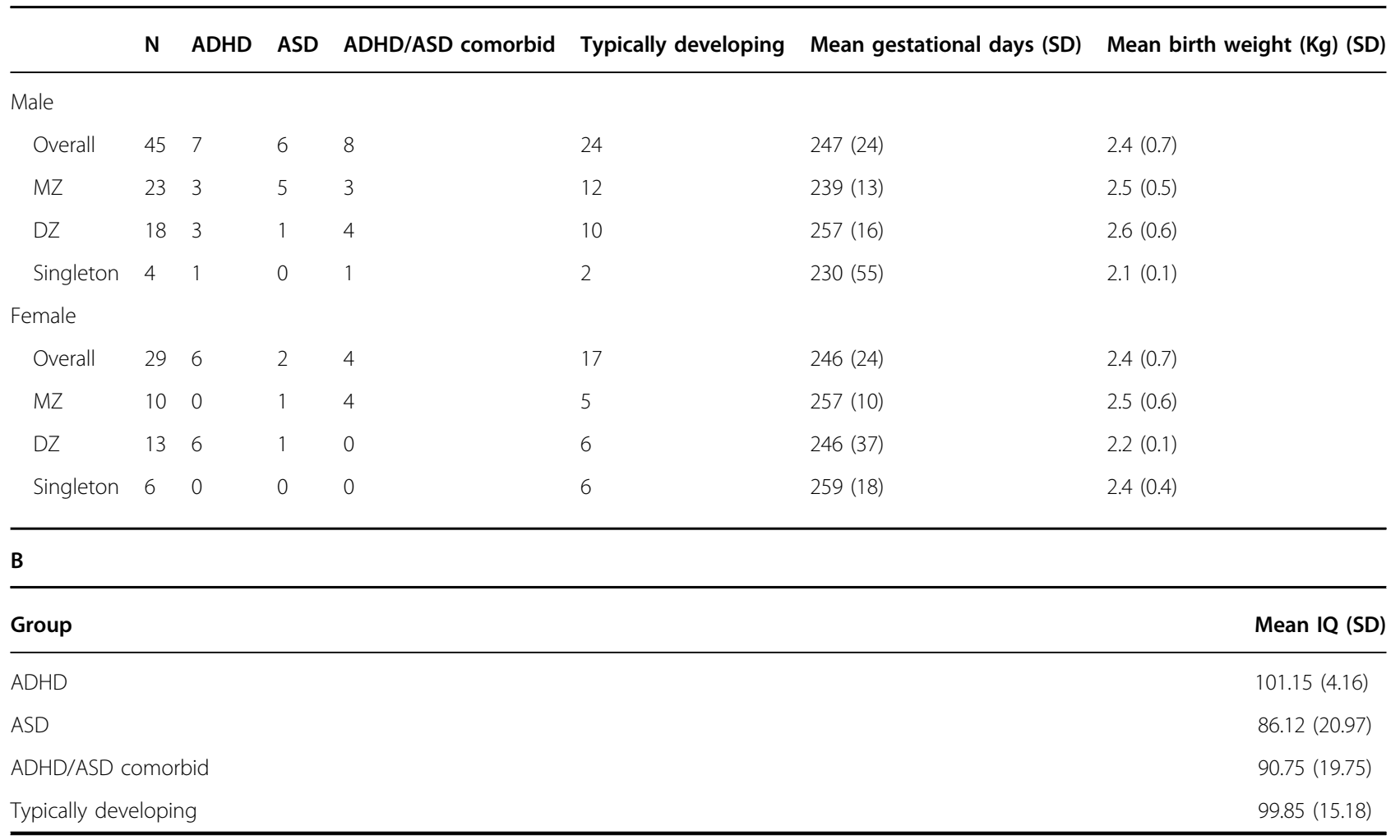


accounts for $\sim 50 \%$ of the whole RATSS cohort of tooth shedding age. The clinical aspect of the study and sample collection were approved by the Swedish National Ethical Review Board. All participants gave informed consent. Analyses were also approved by the Institutional Review Board of the Icahn School of Medicine.

\section{Clinical assessment}

Participants were diagnosed using a consensus process with several experienced clinicians, and according to DSM-5 criteria, endorsed by information from the following standardized instruments: Kiddie Schedule for Affective Disorders and Schizophrenia (K-SADS ${ }^{25}$ ), Diagnostic Interview for ADHD in Adults (DIVA 2.006), Autism Diagnostic Observation Schedule 2nd Edition $\left(\right.$ ADOS- $2^{27}$ ), and Autism Diagnostic Interview- Revised $\left(A D I-R^{28}\right)$. IQ testing was performed with the following measures depending on participant's age and verbal capacities: Wechsler Intelligence Scale for Children-IV $\left(\right.$ WISC-IV ${ }^{29}$ ), Wechsler Adult Intelligence Scale-IV $\left(\right.$ WAIS-IV ${ }^{30}$ ), and the Leiter International Performance Scale-Revised ${ }^{30}$ ).

\section{Laboratory analysis}

Our approach to laser ablation-inductively coupled plasma-mass spectrometry (LA-ICP-MS) tooth metals analysis and assigning developmental times has been detailed elsewhere ${ }^{31,32}$. Briefly, teeth are sectioned vertically along the labio-lingual/buccal-lingual plane and sampled parallel to the dentine-enamel junction from the dentine horn tip towards the tooth cervix. Depending on scan length, each tooth is sampled at 152 locations on average. Temporal information is assigned to sampling points using the neonatal line, a histological feature formed in enamel and dentine at the time of birth, and additional pre- and postnatal incremental markings. A New Wave Research NWR-193 (ESI, USA) laser ablation unit equipped with a $193 \mathrm{~nm}$ ArF excimer laser was connected to an Agilent Technologies 8800 triple-quad ICP-MS (Agilent Technologies, USA). Ablation was carried out under a helium atmosphere which is mixed with argon via Y-piece before introduction to the ICP-MS. Instrument sensitivity (maximum analyte ion counts), oxide formation $\left({ }^{232} \mathrm{Th}^{16} \mathrm{O}^{+} /{ }^{232} \mathrm{Th}^{+},<0.3 \%\right)$ and fractionation $\left({ }^{232} \mathrm{Th}^{+} /{ }^{238} \mathrm{U}^{+}, 100 \pm 5 \%\right)$ were monitored daily using NIST SRM 612 (trace elements in glass). Data were collected as metal to calcium ratios (e.g., ${ }^{208} \mathrm{~Pb}:{ }^{43} \mathrm{Ca}$ ) to control for any variations in the mineral content within a tooth and between samples.

\section{Recurrence quantification analysis}

As previously described in Curtin et al. ${ }^{19,33}$, we used non-linear methods-recurrence quantification analysis (RQA) and cross-recurrence quantification analysis
(CRQA) - to characterize dynamic, cyclical properties of environmental exposures and their metabolism. Briefly, time-series data sampled from teeth were used to construct recurrence plots of single elements or crossrecurrence plots of two elements. We investigated the temporal features generated by recurrence plots, focusing on determinism, mean diagonal length, entropy, and recurrence time. These features measure diagonal line structures, or cyclical events, in recurrence plots. Determinism measures the ratio of diagonal lines (cyclical events) to vertical and/or horizontal lines (non-cyclical events) effectively defining the periodicity of an elemental time-series. Mean diagonal length measures the mean duration of diagonal lines or cycles, and recurrence time measures the interval between these cycles. Entropy captures the complexity of cyclic activity by measuring the variability in cycle lengths. These measures were similarly generated for CRQA, but captured the temporal relationship between two elemental signals. These methods are summarized in Fig. 1 and were processed with the Cross-Recurrence Toolbox v5.16 $6^{34}$ in Matlab v2016b (Mathworks).

\section{Statistical methods}

Prior to applying inferential statistical testing, all variables were evaluated to confirm assumptions of normality in value distributions. Values of \pm 2 SDs were excluded from some variables to meet assumptions of normality. For analyses of recurrence features (RQA/CRQA, described above), linear mixed models were used to test main dichotomous effects of the presence/absence of an ADHD-diagnosis on these features. Twin pairs were modeled as random variables, while also controlling for sex, gestational days, birth weight, and comorbid ASD status. Additional covariates, such as zygosity and IQ, were initially included in model construction but were ultimately excluded as these yielded no improvement in model fit (Akaike Information Criteria, AIC), had no significant effects, or caused no changes in the significance of other covariates included in modeling. False discovery rate (FDR) adjustments, stratified by metal pathway (or cross recurrence), were applied to raw p-values relating to ADHD effects; unless otherwise noted, all reported p-values reflect FDR adjustment. Adjusted p-values less than or equal to 0.05 are reported as significant.

We additionally implemented dimensionality-reduction techniques to investigate the utility of these methods in characterizing neurotypical, ADHD, ASD, and ADHD/ ASD comorbid phenotypes. We first applied principal component analysis (PCA), an unsupervised dimensionality-reduction technique, to investigate relationships among features derived from RQA, and evaluate the efficacy of derived components. Measures derived 


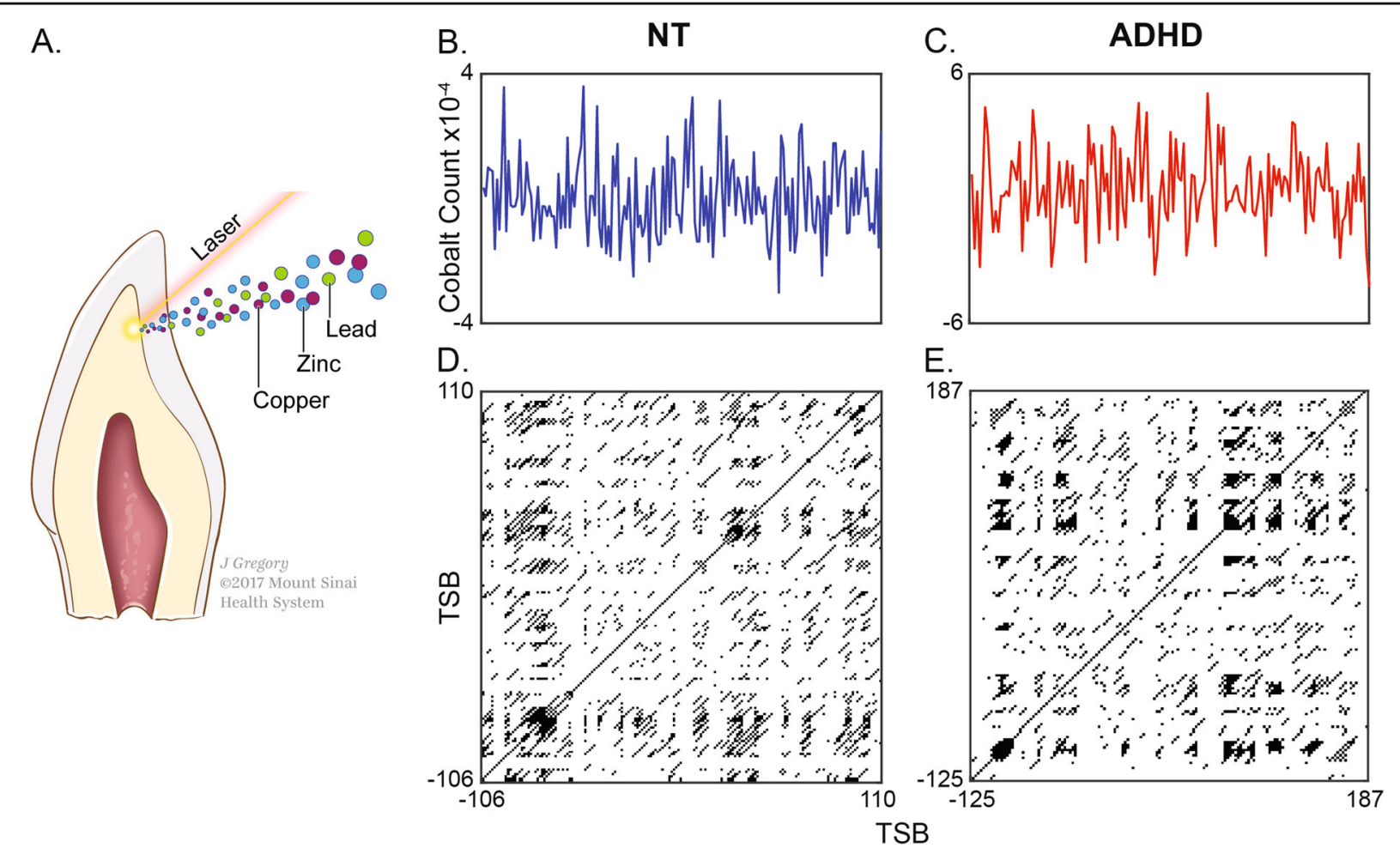

Fig. 1 Overview of study design. a Collected deciduous teeth were analyzed using laser ablation-inductively coupled plasma-mass spectrometry to generate temporal profiles of metal uptake during fetal and postnatal development. $\mathbf{b}$ Example cobalt exposure profile in a typically-developed (TD) control subject ranging from -106 to 110 days since birth. c Example cobalt exposure profile in a subject diagnosed with ADHD ranging from -125 to 187 days since birth. $\mathbf{d}$ Recurrence plot generated from control element trace in panel $\mathbf{b}$. This graphical analytical tool presents cyclical processes as diagonal lines; in recurrence quantification analysis (RQA), the duration (mean diagonal line length, MDL), complexity (entropy), and determinism (proportion of diagonal elements) of cyclical processes are analyzed to characterize dynamical features in elemental time series. e Recurrence plot generated from ADHD case in panel $\mathbf{c}$. The relative abundance of laminar states (black structures) and attenuated diagnoal length indicate reduced periodicity

from RQA of single elements and cross-recurrence (CRQA) analyses, including Determinism, Entropy, Mean Diagonal Length, and Recurrence Time, were centered and scaled for PCA. Following inspection of a scree plot, 15 components were retained for subsequent analyses, as these explained $>80 \%$ of total variance and all estimated eigenvalues were greater than 1 . Linear models were used to evaluate if derived components differed between subjects diagnosed as neurotypical, ASD, ADHD, or comorbid with ASD and ADHD. PCA was implemented in R v 3.5.2 with the factoextra package.

We next complemented the unsupervised analysis described above with a linear discriminant analysis (LDA), a supervised dimensionality-reduction technique, to evaluate the separation of ADHD, ASD, comorbid ASD/ ADHD and neurotypical behavioral phenotypes on the basis of elemental metabolic features extracted via RQA. This analysis simultaneously evaluated all 60 features derived from eight elemental pathways and seven crossrecurrences to separate behavioral diagnoses along a lower dimensional space. Subjects with missing values for any of these features were excluded from this analysis. For each discriminant axis calculated, the correlation between raw RQA features and discriminant scores was calculated to provide a standardized measure of variable loading/ importance as it related to a given axis. Analyses were implemented in R v 3.5.2 with the mass package.

\section{Results \\ Dysregulation of elemental profiles in ADHD}

We first analyzed the cyclical processes of individual elemental profiles in ADHD cases and compared those to neurotypical controls. We found that ADHD cases consistently showed a decrease in overall rhythmicity of metal levels over time, which is measured by determinsim; these effects are summarized per-metal in Supplemental Table 2. In ADHD cases, determinism was reduced in the elements cobalt $(\beta=-0.03, P=0.017)$, lead $(\beta=-0.03, P=0.016)$, and vanadium $(\beta=-0.03, P=0.01)$; and, there was a tendency for copper to be reduced $(\beta=-0.03, P=0.054)$. These results indicate that in ADHD cases there is instability in the cyclic metabolic activity of these 

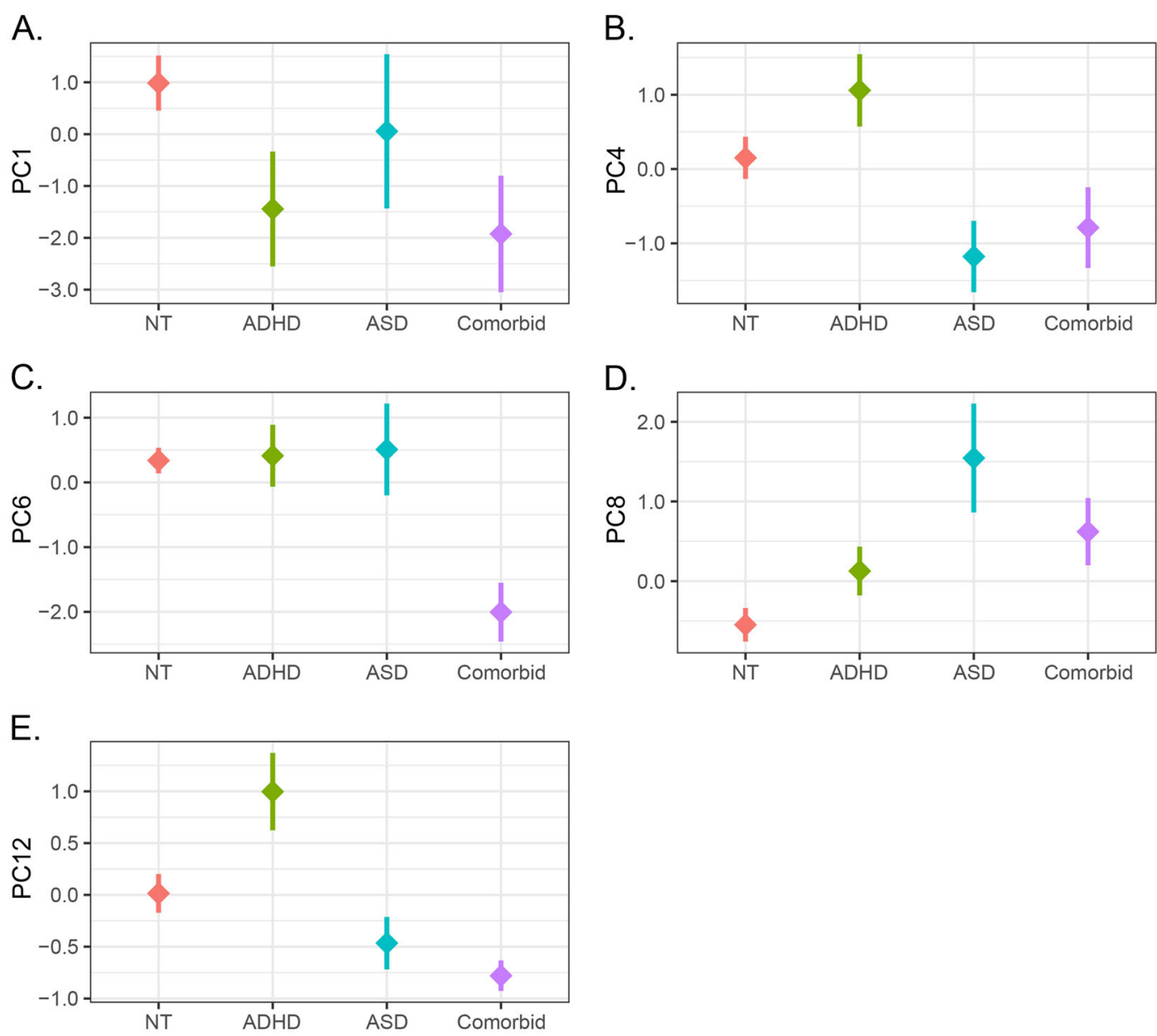

Fig. 2 Principal components underlying elemental metabolism in ASD and ADHD. Panels show mean \pm SEM for component scores along principal components $1,4,6,8$, and 12

elements, relative to what occurs in typically developing twins. We found a significant effect of birth weight in zinc $(\beta=-0.00002, P=0.03)$, but no other significant effects relating to determinism across metals in our covariate adjustments (i.e., sex, gestational days, birth weight, and ASD).

We also saw significant reduction in the duration and complexity of some elemental cycles. In ADHD cases, the duration of elemental cycles, measured by mean diagonal length, was reduced in lead $(\beta=-0.38, P=0.016)$ and vanadium ( $\beta=-0.25, P=0.01)$, compared to neurotypical controls (Supplemental Table 2). Furthermore, entropy (a measure of the variability in cycle lengths) was decreased in cobalt $(\beta=-0.13, P=0.017)$, lead $(\beta=$ $-0.18, P=0.016)$, and vanadium $(\beta=-0.15, P=0.008)$ in ADHD cases (Supplemental Table 2). Again, we found birth weight had a significant effect on zinc entropy $(\beta=$ $-0.0001, P=0.03$ ), but none of the other covariates included in these models had significant effects across elements. Additionally, we found no significant differences in the intervals between cyclical processes (i.e., recurrence time) between cases and controls.

\section{Dysregulation of cross-elemental profiles in ADHD}

Next we tested co-regulation of zinc and other elements, because zinc is known to be a central regulator of other metals and its associated pathways have been implicated in protection against toxicants, e.g., lead ${ }^{35}$, and we previously found zinc dysregulation was related to $\mathrm{ASD}^{19}$. Decreased determinism was not a characteristic feature of zinc-element interactions in ADHD cases, with the exception of zinc-copper $(\beta=-0.03, P=0.024)$ (Supplemental Table 3).

The duration of periodic cycles in ADHD cases was disrupted in three out of seven zinc-element profiles. However, after FDR correction, only one of these pathways was significantly different between ADHD cases and neurotypical controls. Mean diagonal length was significantly shorter in zinc-vanadium cross recurrence $(\beta=$ $-0.24, P=0.036)$, and tended to be reduced in zinc-tin 


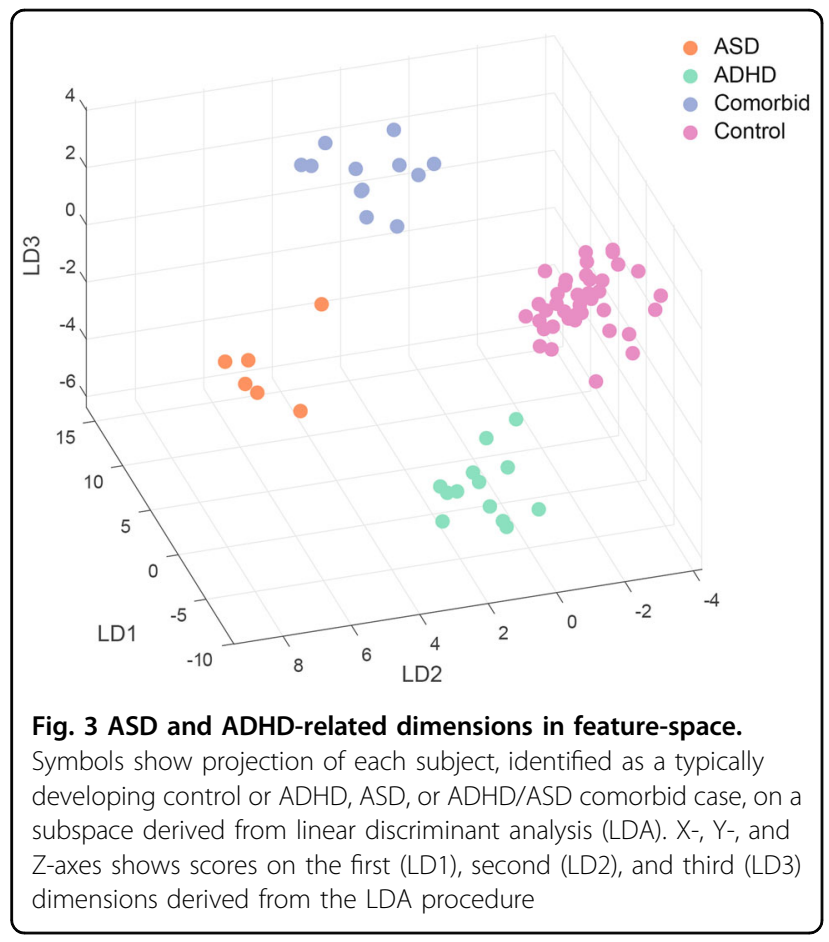

cycles $(\beta=-0.33, P=0.058)$ and zinc-calcium cycles $(\beta=-0.23$, FDR-adjusted $P=0.15$, raw $p=0.04$ ) (Supplemental Table 3). Entropy was also disrupted in ADHD cases in zinc-vanadium $(\beta=-0.11, P=0.036)$ (Supplemental Table 3). Zinc-vanadium $(\beta=-0.71, P=0.036)$ was the only element-pair to show differences in recurrence time, reflecting the interval durations between cyclic periods (Supplemental Table 3).

\section{Phenotypic signatures of ADHD, ASD, ADHD/ASD combined vs neurotypical cases}

We have previously reported how elemental regulation in ASD differs from typical development ${ }^{19,24}$. While the analyses described thus far focused on contrasting individuals with $\mathrm{ADHD}$ from neurotypical controls while controlling for ASD, here we sought to leverage the highdimensional features derived from multiple pathways to distinguish signatures unique to these diagnoses. We applied principal component analysis (PCA) and linear discriminant analysis (LDA) to leverage both unsupervised and supervised dimensionality-reduction techniques to achieve this goal.

Our initial analysis with PCA identified 15 components with eigenvalues exceeding 1, accounting for $82.79 \%$ of total variance across RQA/CRQA features. Among this subset of components, we compared component scores among participants, and identified 5 axes relating to neurobehavioral phenotypes, as shown in Fig. 2. Along the first principal component, we identified a significant reduction in component scores relative to neurotypical controls both for ADHD subjects $(\beta=-2.42, P=0.04)$ and comorbid cases $(\beta=-2.91, P=0.02)$, as shown in Fig. 2 (panel A). We also identified factors relevant to ADHD along the $12^{\text {th }}$ principal component, which was significant elevated in ADHD participants $(\beta=0.98, P=0.006)$, but was depressed in subjects comorbid for ASD/ADHD $(\beta=$ $-0.79, P=0.03)$. In principal components 4 and 8 , we found effects associated with ASD diagnosis, with significantly reduced scores on principal component 4 ( $\beta=$ $-1.33, P=0.04)$, and elevated scores on component 8 for ASD $(\beta=2.09, P<0.0001)$ and comorbid cases $(\beta=1.19$, $P=0.01)$. Dysregulation along principal component 6 was specific to comorbid ASD/ADHD cases, with significant reductions relative to neurotypical controls $(\beta=-2.34$, $P<0.0001)$. Supplementary Fig. 1 provides the variable loadings associated with each component.

We complemented this approach with a linear discriminant analysis (LDA) to visualize separation of these classes across a low-dimensional feature space. This projection, shown in Fig. 3, emphasizes the separation of neurodevelopmental phenotypes by elemental metabolic features. In Fig. 4, we show how the separation of each neurodevelopmental phenotype manifests across linear discriminant axes (A1, B1, C1), and the standardized loadings of RQA features on these dimensions (A2, B2, C2). The first axis, LD1, primarily captures the separation of comorbid ADHD/ASD cases from neurotypical, ASD, and ADHD cases (Fig. 4, A1), with zinc-copper cycle complexity (entropy) and duration (mean diagonal length), and zinc-lead determinism (rhythmicity) contributing most to this separation (Fig. 4, A2). Similarly, LD2 primarily captures the separation of ASD and ADHD cases (Fig. 4, B1), though along this axis zinc-tin mean diagonal length, zinc-copper recurrence time, and lithium recurrence time also contribute to this separation, in addition to the features described above. LD3 captures the separation of ADHD cases from all other phenotypes (Fig. 4, C1), primarily driven by copper and cobalt determinism, vanadium entropy and determinism, and lithium entropy and mean diagonal length, among other features. The integration of dynamical features extracted from multiple elemental pathways thus identifies a signature associated with each behavioral phenotype. The complete listings of the relative contribution of each feature to each discriminative axis are provided in Fig. 4 (A2, B2, C2).

\section{Discussion}

Our results show that cyclical processes involved in the metabolism of essential elements and toxic metals during fetal and early postnatal development differ significantly in ADHD and neurotypical development. We used tooth matrix biomarkers to measure detailed pre- and postnatal temporal profiles of lead, cobalt, zinc, vanadium, and other 


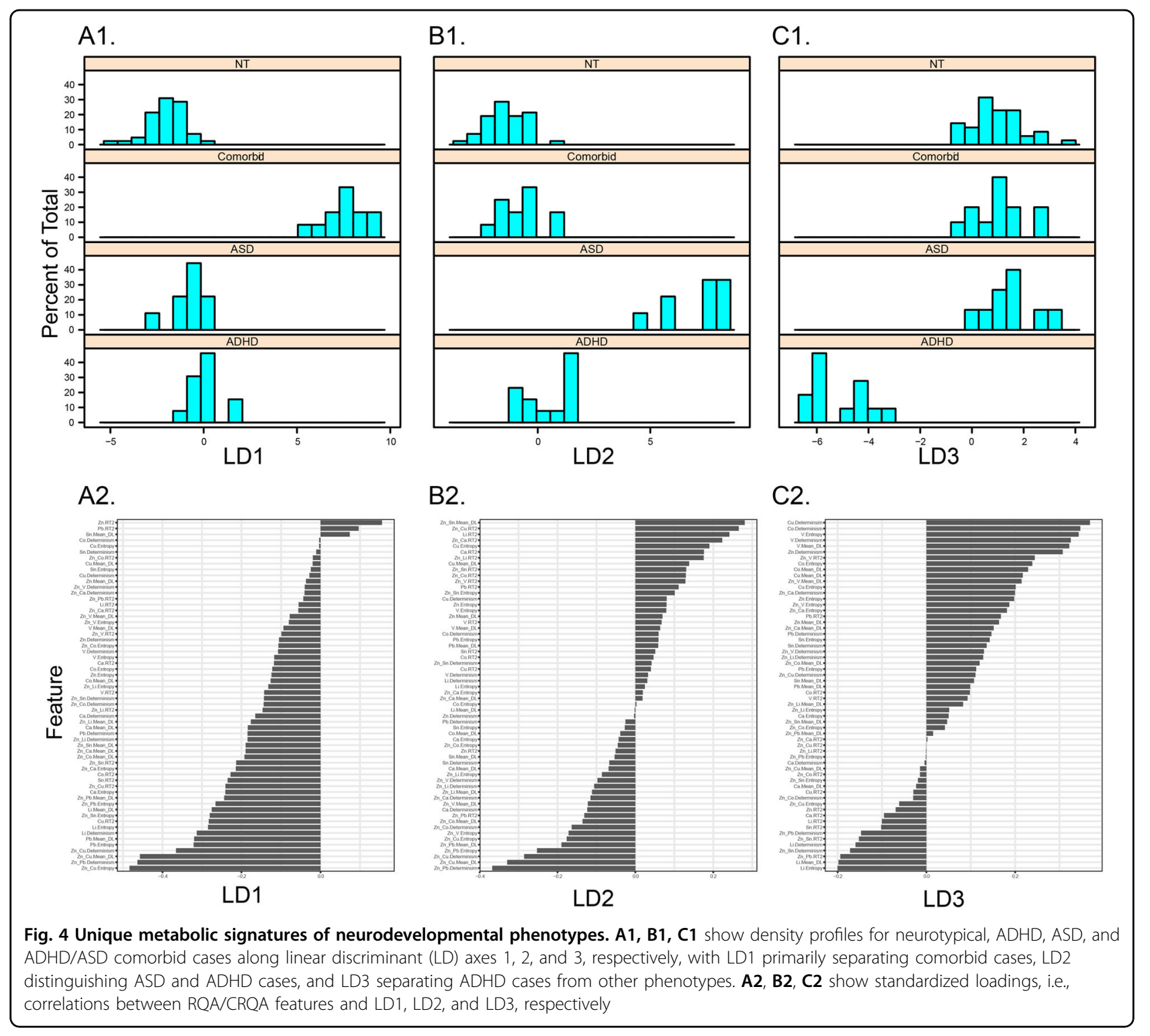

elements, and tested the hypothesis that alterations of metal metabolism cycles are associated with ADHD. Overall, we found that the stability, duration, and complexity of cyclical processes were reduced in ADHD cases compared to controls. These findings jointly suggest that the metabolism of essential and toxic elements is affected in ADHD. Critically, we were able to identify distinct cyclical properties specific to ADHD alone, as well as shared elemental pathways common to both ADHD and ASD.

In ADHD, we saw decreased cycle stability (determinism), duration (mean diagonal length), and complexity (entropy) in cobalt, copper, lead, zinc, and vanadium exposure profiles. Previous studies have shown that increased levels of lead are correlated with the severity of ADHD symptoms ${ }^{7}$, specifically, rather than neurodevelopmental conditions like ASD in general. Interestingly, we were not only able to identify distinct elemental signatures for those with either ADHD or ASD, but also in combined behavioral presentations. This finding at the molecular level is in broad agreement with clinical features of these conditions where significant overlap is observed in symptomatology ${ }^{36-38}$.

Our findings are also bolstered by human and animal studies where exposure to environmental chemicals altered pathways relevant to the symptoms observed in $\mathrm{ADHD}^{39-41}$. A handful of studies describe the possible roles for zinc in ADHD. Zinc is an essential element involved in dopamine pathways that are assumed to be involved in ADHD etiology. Zinc deficiency has been hypothesized to cause dysfunction of the dopamine 
transporter ${ }^{42}$, impaired dopaminergic transmission ${ }^{41}$ and modulation of melatonin, a regulator of dopamine function and potential pathway of amphetamine treatment of $\mathrm{ADHD}^{43}$ or ADHD-related sleep problems ${ }^{44}$. Several enzymes believed to have an essential role in the neurophysiology of ADHD are dependent on copper ${ }^{45}$. A dysregulation of zinc or copper may also increase susceptibility to oxidative damage of tissues or oxidative stress of the brain by damaging antioxidant defenses, a possible pathophysiology of ADHD ${ }^{41,46,47}$. Excess copper may promote the oxidation of dopamine and its metabolite salsolinal, leading to the degeneration of dopaminergic neurons ${ }^{46}$. Lead may contribute to dopaminergic dysfunction ${ }^{48}$ and disrupt the blood-brain barrier ${ }^{49}$.

Our study is limited by a small sample size, although it was sufficient to distinguish significant differences in cyclical metal properties between ADHD, ASD, ADHD/ ASD combined, and neurotypical development. As a consequence of this, it was not possible to implement robust cross-validation procedures, and these results should therefore not be interpreted as a generalizable algorithm for predictive phenotyping or diagnostic assessment. Nonetheless, the utility of both supervised and unsupervised methods in separating neurodevelopmental phenotypes, and the general overlap in features that discriminate ASD \& ADHD, suggest that elemental dynamics, particularly relating to zinc periodicity, may provide a powerful signature for future studies to leverage for predictive phenotyping. The differentiation of behavioral phenotypes including ADHD, ASD and their combined presentation from elemental metabolic features further emphasizes the significance of these processes in neurodevelopment, and the potential future applications of these methods in a broader sample. The use of twins in our study allowed us to control for underlying genetic factors and to increase our power to detect differences between diagnostic groups. However, further work is needed in non-twin participants to confirm and extend our results. Organic environmental chemicals may also play a role in ADHD, a topic not addressed in our study.

Studies of environmental exposures and ADHD have primarily focused on the magnitude of exposures as determined by concentrations in blood, urine or other biological matrices. By analyzing dynamical processes in elemental exposure profiles, we have uncovered differences in elemental metabolism that would not be apparent in measures of exposure intensity alone. Furthermore, our findings have implications for early detection of ADHD, ASD, and ADHD/ASD combined cases because the signatures we have observed are present prenatally and in early postnatal development. Pathways we have identified, once validated, may also offer targets for prevention of toxic exposures and early intervention in the form of modification of elemental metabolism towards a healthy molecular phenotype.

\section{Acknowledgements \\ We express our sincere gratitude to all twins and parents who have} participated in this research. We also thank the RATSS team at the Center of Neurodevelopmental Disorders at Karolinska Institutet (KIND) for their valuable contribution to the work presented in this study. Genotyping was performed by the SNP\&SEQ Technology Platform in Uppsala (www.genotyping.se). The facility is part of the National Genomics Infrastructure (NGI) Sweden and Science for Life Laboratory. The SNP\&SEQ Platform is also supported by the Swedish Research Council and the Knut and Alice Wallenberg Foundation. The study was funded by the Swedish Research Council, Vinnova, Formas, FORTE, the Swedish Brain foundation (Hjärnfonden), Stockholm Brain Institute, Autism and Asperger Association Stockholm, Queen Silvia's Jubilee Fund, Solstickan Foundation, PRIMA Child and Adult Psychiatry, the Pediatric Research

Foundation at Astrid Lindgren Children's Hospital, the Swedish Foundation for Strategic Research, Jerring Foundation, the Swedish Order of Freemasons, Kempe-Carlgrenska Foundation, Sunnderdahls Handikappsfond, The Jeansson Foundation, and EU-AIMS (European Autism Intervention), with support from the Innovative Medicines Initiative Joint Undertaking (grant agreement no.

115300), the resources of which are composed of financial contributions from the European Union's Seventh Framework Programme (grant FP7/2007-2013), from the European Federation of Pharmaceutical Industries and Associations companies' in-kind contributions, and from Autism Speaks. It was also supported by a new IMI initiative-EU AIMS-2-TRIALS. E.D.S. group was supported by the National Institute of Environmental Health Sciences research grants (DP2ES025453, R21ES023604, R01ES026033, P30ES023515,

U2CES026561).

\section{Author details}

${ }^{1}$ Department of Environmental Medicine and Public Health, Icahn School of Medicine at Mount Sinai, One Gustave L Levy Place, Box 1057, New York, NY 10029, USA. ${ }^{2}$ Karolinska Institutet Center of Neurodevelopmental Disorders (KIND), Centre for Psychiatry Research, Department of Women's and Children's Health, Karolinska Institutet, \& Stockholm Health Care Services, Stockholm County Council, Stockholm, Sweden. ${ }^{3}$ Department of Neuroscience, Child and Adolescent Psychiatry Unit, Uppsala University, Uppsala, Sweden. ${ }^{4}$ Department of Molecular Medicine and Surgery, Center for Molecular Medicine, Karolinska Institutet, Stockholm, Sweden. ${ }^{5}$ Curtin Autism Research Group, Essential

Partner Autism CRC, School of Occupational Therapy, Social Work and Speech Pathology, Curtin University, Perth, WA, Australia

Conflict of interest

The authors declare that they have no conflict of interest.

\section{Publisher's note}

Springer Nature remains neutral with regard to jurisdictional claims in published maps and institutional affiliations.

Supplementary Information accompanies this paper at (https://doi.org/ 10.1038/s41398-019-0567-6).

Received: 26 March 2019 Accepted: 30 July 2019

Published online: 25 September 2019

\section{References}

1. Polanczyk, G., de Lima, M. S., Horta, B. L., Biederman, J. \& Rohde, L. A. The worldwide prevalence of ADHD: a systematic review and metaregression analysis. Am. J. Psychiatry 164, 942-948 (2007).

2. Simon, V., Czobor, P., Balint, S., Meszaros, A. \& Bitter, I. Prevalence and correlates of adult attention-deficit hyperactivity disorder: meta-analysis. Br. J. Psychiatry 194, 204-211 (2009).

3. Polanska, K., Jurewicz, J. \& Hanke, W. Review of current evidence on the impact of pesticides, polychlorinated biphenyls and selected metals on 
attention deficit/hyperactivity disorder in children. Int J. Occup. Med. Env 26, 16-38 (2013).

4. Faraone, S. V. et al. Molecular genetics of attention-deficit/hyperactivity disorder. Biol. Psychiatry 57, 1313-1323 (2005).

5. Brikell, I., Kuja-Halkola, R. \& Larsson, H. Heritability of attention-deficit hyperactivity disorder in adults. Am. J. Med. Genet. Part B, Neuropsychiatr. Genet.: Off. Publ. Int. Soc. Psychiatr. Genet. 168, 406-413 (2015).

6. Bölte, S., Poustka, L. \& Geurts, H. in Oxford Textbook of Attention Deficit Hyperactivity Disorder. (eds Tobias Banaschewski, David Coghill, \& Alessandro Zuddas) (Oxford University Press, Oxford, UK, 2018).

7. Kern, J. K, Geier, D. A., Sykes, L. K., Geier, M. R. \& Deth, R. C. Are ASD and ADHD a continuum? A comparison of pathophysiological similarities between the disorders. J. Atten. Disord. 19, 805-827 (2015).

8. Froehlich, T. E. et al. Update on environmental risk factors for attention-deficit/ hyperactivity disorder. Curr. Psychiat Rep. 13, 333-344 (2011).

9. Park, J. H. et al. Blood lead concentrations and attention deficit hyperactivity disorder in Korean children: a hospital-based case control study. BMC Pediatrics 16, 156 (2016).

10. Goodlad, J. K. Marcus, D. K. \& Fulton, J. J. Lead and Attention-Deficit/Hyperactivity Disorder (ADHD) symptoms: A meta-analysis. Clin. Psychol. Rev. 33, 417-425 (2013).

11. Farias, A. C. et al. Manganese in children with attention-deficit/hyperactivity disorder: relationship with methylphenidate exposure. J. Child Adolesc. Psychopharmacol. 20, 113-118 (2010).

12. Wang, Y., Huang, L., Zhang, L., Qu, Y. \& Mu, D. Iron status in attention-deficit/ hyperactivity disorder: a systematic review and meta-analysis. PLOS ONE. https://doi.org/10.1371/journal.pone.0169145 (2017).

13. Polańska, K., Jurewicz, J. \& Hanke, W. Review of current evidence on the impact of pesticides, polychlorinated biphenyls and selected metals on attention deficit/hyperactivity disorder in children. Int. J. Occup. Med. Environ. Health 26, 16-38 (2013)

14. Boucher, O. et al. Prenatal methylmercury, postnatal lead exposure, and evidence of attention deficit/hyperactivity disorder among inuit children in Arctic QuÃ@bec. Environ. Health Perspect. 120, 1456-1461 (2012).

15. Cheuk, D. K. L. \& Wong, V. Attention-deficit hyperactivity disorder and blood mercury level: a case-control study in chinese children. Neuropediatrics 37, 234-240 (2006).

16. Oner, O. et al. Effects of zinc and ferritin levels on parent and teacher reported symptom scores in attention deficit hyperactivity disorder. Child Psychiatry Hum. Dev. 41, 441-447 (2010).

17. Arnold, L. E. et al. Serum zinc correlates with parent- and teacher- rated inattention in children with attention-deficit/hyperactivity disorder. J. Child Adolesc. Psychopharmacol. 15, 628-636 (2005).

18. Viktorinova, A. et al. Changed plasma levels of zinc and copper to zinc ratio and their possible associations with parent- and teacher-rated symptoms in children with attention-deficit hyperactivity disorder. Biol. Trace Elem. Res. 169 1-7 (2016).

19. Curtin, P. et al. Dynamical features in fetal and postnatal zinc-copper metabolic cycles predict the emergence of autism spectrum disorder. Sci. Adv. $\mathbf{4}$, eaat1293 (2018)

20. Eckmann, J., Oliffson, S. \& Ruelle, D. Recurrence plots of dynamical systems. Europhys. Lett. 4, 973-977 (1987).

21. Marwan, N. \& Kurths, J. Nonlinear analysis of bivariate data with cross recurrence plots. Phys. Lett. A 302, 299-307 (2002).

22. Marwan, N., Romano, M. C., Thiel, M. \& Kurths, J. Recurrence plots for the analysis of complex systems. Phys. Rep. 438, 237-329 (2007).

23. Bolte, S. et al. The Roots of Autism and ADHD Twin Study in Sweden (RATSS). Twin Res Hum. Genet 17, 164-176 (2014).

24. Arora, M. et al. Fetal and postnatal metal dysregulation in autism. Nat. Commun. https://doi.org/10.1038/ncomms15493 (2017)

25. Kaufman, J. et al. Schedule for Affective Disorders and Schizophrenia for School-Age Children-Present and Lifetime Version (K-SADS-PL): initial reliability and validity data. J. Am. Acad. Child Adolesc. Psychiatry 36, 980-988 (1997)

26. Kooij, J. Diagnostic Interview for ADHD in Adults 2.0 (DIVA). (2010).
27. Lord, C., Rutter, M., DiLavore, P. Risi, S., Gotham, K., \& Bishop, S. Autism Diagnostic Observation Schedule - 2nd Edition (ADOS-2). (Western Psychological Services, Torrance, CA, 2012).

28. Rutter, M., Le Couteur, A., \& Lord, C. The Autism Diagnostic Interview - Revised (ADI-R). (Western Psychological Services, Los Angeles, CA, 2003).

29. Wechsler, D. WISC-N Wechsler Intelligence Scale for children 4th edition: Technical and interpretive manual. (The Psychological Corporation, Weshsler Intelligence Scale for children, San Antonia, TX, 2003).

30. Roid, G. H., \& Miller, L. J. Leiter international performance scale-revised: Examiner's manual. (Stoelting, Weshsler Intelligence Scale for adults, Wood Dale, IL, 1997).

31. Arora, M. \& Austin, C. Teeth as a biomarker of past chemical exposure. Curr. Opin. Pediatr. 25, 261-267 (2013).

32. Austin, C. et al. Barium distributions in teeth reveal early-life dietary transitions in primates. Nature 498, 216-219 (2013).

33. Curtin, P. et al. Recurrence quantification analysis to characterize cyclical components of environmental elemental exposures during fetal and postnatal development. PLoS ONE 12, e0187049 (2017).

34. Marwan, N. Cross recurrence plot toolbox for Matlab, Ver. 5.22 (R32.1), http.// tpcsy.pik-postdam.de/CRPtoolbox (2002).

35. Upadhyay, A. K., Mathur, R., Bhadauria, M. \& Nirala, S. K. Therapeutic influence of zinc and ascorbic acid against lead induced biochemical alterations. Therapie 64, 383-388 (2009).

36. Simonoff, E. et al. Psychiatric disorders in children with autism spectrum disorders: prevalence, comorbidity, and associated factors in a population-derived sample. J. Am. Acad. Child Adolesc. Psychiatry 47, 921-929 (2008)

37. Hartman, C. A., Geurts, H. M., Franke, B., Buitelaar, J. K. \& Rommelse, N. N. J. Changing ASD-ADHD symptom co-occurrence across the lifespan with adolescence as crucial time window: Illustrating the need to go beyond childhood. Neurosci. Biobehav. Rev. 71, 529-541 (2016).

38. Holtmann, M., Bolte, S. \& Poustka, F. Attention deficit hyperactivity disorder symptoms in pervasive developmental disorders: association with autistic behavior domains and coexisting psychopathology. Psychopathology 40, 172-177 (2007)

39. Carpenter, D. O., Hussain, R. J., Berger, D. F., Lombardo, J. P. \& Park, Y. Y. Electrophysiologic and behavioral effects of perinatal and acute exposure of rats to lead and polychlorinated biphenyls. Environ. Health Persp 110, 377-386 (2002).

40. Lehohla, M., Russell, V. \& Kellaway, L. NMDA-stimulated Ca2+ uptake into barrel cortex slices of spontaneously hypertensive rats. Metab. Brain Dis. 16, 133-141 (2001).

41. Scassellati, C., Bonvicini, C., Faraone, S. V. \& Gennarelli, M. Biomarkers and attention-deficit/hyperactivity disorder: a systematic review and meta-analyses. J. Am. Acad. Child Psy 51, 1003-1019 (2012).

42. Lepping, P. \& Huber, M. Role of zinc in the pathogenesis of attention-deficit hyperactivity disorder: implications for research and treatment. CNS Drugs $\mathbf{2 4}$ 721-728 (2010)

43. Sandyk, R. Zinc deficiency in attention-deficit hyperactivity disorder. Int J. Neurosci. 52, 239-241 (1990).

44. Coogan, A. N. \& McGowan, N. M. A systematic review of circadian function, chronotype and chronotherapy in attention deficit hyperactivity disorder. Atten. Defic. Hyperact Disord. 9, 129-147 (2017).

45. Llanos, R. M. \& Mercer, J. F. The molecular basis of copper homeostasis copperrelated disorders. DNA Cell Biol. 21, 259-270 (2002).

46. Yu, W. R. Jiang, H., Wang, J. \& Xie, J. X. Copper (Cu2+) induces degeneration of dopaminergic neurons in the nigrostriatal system of rats. Neurosci. Bull. 24, 73-78 (2008)

47. Lopresti, A. L. Oxidative and nitrosative stress in ADHD: possible causes and the potential of antioxidant-targeted therapies. Atten. Defic. Hyperact Disord. 7, 237-247 (2015).

48. Gedeon, Y., Ramesh, G. T., Wellman, P. J. \& Jadhav, A. L. Changes in mesocorticolimbic dopamine and D1/D2 receptor levels after low level lead exposure: a time course study. Toxicol. Lett. 123, 217-226 (2001).

49. Lidsky, T. I. \& Schneider, J. S. Lead neurotoxicity in children: basic mechanisms and clinical correlates. Brain 126, 5-19 (2003). 